\title{
Iterative Multi-User Detection for OFDM Using Biased Mutation Assisted Genetic Algorithms
}

\author{
M. Jiang and L. Hanzo ${ }^{1}$ \\ School of ECS, Univ. of Southampton, SO17 1BJ, UK. \\ Tel: +44-703-593 125, Fax: +44-703-593 045 \\ Email: ${ }^{1}$ lh@ecs.soton.ac.uk, http://www-mobile.ecs.soton.ac.uk
}

\begin{abstract}
Space Division Multiple Access (SDMA) aided Orthogonal Frequency Division Multiplexing (OFDM) systems assisted by efficient Multi-User Detection (MUD) techniques have recently attracted intensive research interests. As expected, Maximum Likelihood (ML) detection was found to attain the best performance, although this was achieved at the cost of a high computational complexity. Forward Error Correction (FEC) schemes such as Turbo Trellis Coded Modulation (TTCM) can be efficiently amalgamated with SDMA-OFDM systems for the sake of improving the achievable performance without bandwidth expansion. In this contribution, a MMSE-aided Iterative GA (IGA) MUD is proposed for employment in a TTCM-assisted SDMA-OFDM system, which is capable of achieving a similar performance to that attained by its optimum ML-aided counterpart at a significantly lower complexity, especially at high user loads. Moreover, when the proposed novel Biased Q-function Based Mutation (BQM) scheme is employed, the IGA-aided system's performance can be further improved by achieving an $E_{b} / N_{0}$ gain of about $6 \mathrm{~dB}$ in comparison to the TTCM-aided MMSE-SDMA-OFDM benchmarker system both in low- and high-throughput modem scenarios, respectively, while still maintaining a modest complexity.
\end{abstract}

\section{INTRODUCTION}

Space Division Multiple Access (SDMA) based Orthogonal Frequency Division Multiplexing (OFDM) [1] communication invoking MultiUser Detection (MUD) techniques has recently attracted intensive research interests. In SDMA Multi-Input-Multi-Output (MIMO) systems the transmitted signals of $L$ simultaneous uplink mobile users - each equipped with a single transmit antenna - are received by the $P$ different receiver antennas of the Base Station (BS). At the BS the individual users' signals are separated by Multi-User Detectors (MUDs) with the aid of the user-specific spatial signature constituted by their channel transfer functions or, equivalently, Channel Impulse Responses (CIRs). In the literature, Maximum Likelihood (ML) detection [1,2] was found to give the best performance, although this was achieved at the cost of a dramatically increased computational complexity, especially in the context of a high number of users and higher-order modulation schemes. By contrast, Minimum Mean-Square Error (MMSE) $[1,2]$ detection exhibits a low complexity, while suffering from a performance loss.

Furthermore, the achievable performance can be significantly improved, if Forward Error Correction (FEC) schemes are incorporated into the SDMA system. Various Coded Modulation (CM) [3] schemes have attracted intensive research interests, since they are capable of achieving a substantial coding gain without bandwidth expansion. It was shown in [4] that Turbo Trellis Coded Modulation (TTCM) [3] generally provides the best performance in the family of $\mathrm{CM}$ schemes in the specific context of the SDMA-OFDM system investigated.

Genetic Algorithms (GAs) [5] have been applied to a number of problems, such as machine learning and modelling adaptive processes. Furthermore, GA-based multiuser detection has been proposed for Code Division Multiple Access (CDMA) systems [6]. Recently, GAs have

Acknowledgements: The work reported in this paper has formed part of the Wireless Enabling Techniques work area of the Core 3 Research Programme of the Virtual Centre of Excellence in Mobile and Personal Communications, Mobile VCE, www.mobilevce.com, whose funding support, including that of EPSRC, is gratefully acknowledged. Fully detailed technical reports on this research are available to Industrial Members of Mobile VCE. also been employed in OFDM systems $[7,8]$ for achieving a nearoptimum performance. Against this background, the novel contribution of this paper is that we propose a MMSE-assisted Iterative GA (IGA) MUD invoking a technique referred to here as the Biased $Q$-function Based Mutation (BQM) designed for improving the performance of a TTCM-aided multi-user SDMA-OFDM system. Our simulation results show that the proposed MMSEIGA assisted TTCM-SDMA-OFDM system is capable of achieving an $E_{b} / N_{0}$ gain of about $6 d B$ in comparison to the TTCMassisted MMSE-SDMA-OFDM benchmarker system both in lowand high-throughput modem scenarios, such as 4QAM and 16QAM, respectively, while maintaining a substantially lower complexity than that imposed by the optimum ML MUD.

The structure of this paper is as follows. The SDMA MIMO channel model is described in Section 2.1, while the overview of the proposed TTCM-aided SDMA-OFDM system using the MMSE-IGA MUD is given in Section 2.2, followed by the introduction of the novel BQM scheme of Section 3. Our simulation results are provided in Section 4 , while Section 5 concludes our findings.

\section{SYSTEM MODEL}

\subsection{SDMA MIMO Channel Model}

In SDMA uplink systems, each of the $L$ simultaneous mobile users employs a single transmit antenna, while the BS's receiver exploits $P$ antennas. At the $k^{t h}$ subcarrier of the $n^{t h}$ OFDM symbol received by the $P$-element receiver antenna array we have the received complex signal vector $\mathbf{x}[n, k]$, which is constituted by the superposition of the independently faded signals associated with the $L$ mobile users and contaminated by the Additive White Gaussian Noise (AWGN), expressed as:

$$
\mathbf{x}=\mathbf{H} \mathbf{s}+\mathbf{n},
$$

where the $(P \times 1)$-dimensional vector $\mathbf{x}$, the $(L \times 1)$-dimensional vector $\mathbf{s}$ and the $(P \times 1)$-dimensional vector $\mathbf{n}$ are the received, transmitted and noise signals, respectively. Here we have omitted the indices $[n, k]$ for each vector for the sake of notational convenience. Specifically, the vectors $\mathbf{x}, \mathrm{s}$ and $\mathrm{n}$ are given by:

$$
\begin{aligned}
\mathbf{x} & =\left(x_{1}, x_{2}, \ldots, x_{P}\right)^{T}, \\
\mathbf{s} & =\left(s^{(1)}, s^{(2)}, \ldots, s^{(L)}\right)^{T}, \\
\mathbf{n} & =\left(n_{1}, n_{2}, \ldots, n_{P}\right)^{T} .
\end{aligned}
$$

The $(P \times L)$-dimensional matrix $\mathbf{H}$ contains the frequency-domain channel transfer functions (FD-CHTF) of the $L$ users and is given by:

$$
\mathbf{H}=\left(\mathbf{H}^{(1)}, \mathbf{H}^{(2)}, \ldots, \mathbf{H}^{(L)}\right),
$$

where $\mathbf{H}^{(l)}(l=1, \ldots, L)$ is the vector of the FD-CHTFs associated with the transmission paths from the $l^{\text {th }}$ user's transmit antenna to each of the $P$-element receiver antenna array, which is expressed:

$$
\mathbf{H}^{(l)}=\left(H_{1}^{(l)}, H_{2}^{(l)}, \ldots, H_{P}^{(l)}\right)^{T}, \quad l=\{1, \ldots, L\},
$$

where $H_{p}^{(l)}(l=1, \ldots, L ; p=1, \ldots, P)$ associated with different user/receiver pairs are assumed to be independent. 
Figure 1: Schematic of the MMSE-IGA MUD assisted multi-user SDMA-OFDM uplink system.

\subsection{System Overview}

In Figure 1, we present the schematic of the proposed MMSE-IGA MUD aided SDMA-OFDM uplink system. At the transmitter end, as seen at the top of Figure 1, the information bit sequences of the geographically separated $L$ simultaneous mobile users are forwarded to the TTCM [3] encoders, where they are encoded into symbols. The encoded signals $s^{(l)}(l=1, \ldots, L)$ are then forwarded to the OFDMrelated Inverse Fast Fourier Transform (IFFT) based modulator, which converts the frequency-domain signals to the time-domain modulated OFDM symbols. The OFDM symbols are then transmitted by the independent Mobile Stations (MSs) to the BS over the SDMA MIMO channel. Then each element of the receiver antenna array shown at the bottom of Figure 1 receives the superposition of the transmitted signals faded and contaminated by the channel and performs Fast Fourier Transform (FFT) based OFDM demodulation. The demodulated outputs $x_{p}(p=1, \ldots, P)$ seen in Figure 1 are forwarded to the proposed MMSE-assisted IGA MUD for separating the different users' signals. The separated signals $\hat{s}^{(l)}(l=1, \ldots, L)$, namely the estimated versions of the transmitted signals, are then independently decoded by the TTCM decoders of Figure 1.

Figure 2: Structure of the MMSE-initialized IGA MUD used at the BS shown in Figure 1.

The detailed structure of the MMSE-IGA MUD is illustrated in Figure 2. As portrayed in Figure 2, the received length- $P$ symbol vector $\mathrm{x}$ of Equation 2 is first detected by the MMSE MUD, which outputs the $L$ MMSE-detected symbols $\hat{s}_{\text {MMSE }}^{(l)}(l=1, \ldots, L)$ of the $L$ users, and forwards them to $L$ number of independent TTCM decoders. The TTCM-decoded $L$-symbol vector, which is more reliable than the MMSE MUD's output, is then fed into the concatenated GA MUD for assisting the creation of the initial population. Then the genetically enhanced output symbol vector $\hat{\mathbf{s}}_{\mathrm{GA}}$, which may be expected to become more reliable, will be fed back to the TTCM decoders in order to further improve the signal's quality, invoking a number of iterations. Following the last iteration, the final GA solution will be decoded by the TTCM decoders, and the hard-decision version of the estimated information bits of the $L$ independent users is forwarded to the output, which is only enabled at the final iteration by the switch seen in Figure 2.

Figure 3: The 2D optimization provided by the MMSE-IGA MUD of Figure 2 . The square brackets $[\cdot]$ denote the subcarrier indices in the TTCM-coded frame of length $N$.

Therefore, two improvements have been achieved by the MMSEIGA MUD. Firstly, a more accurate initial knowledge of the transmitted signals, namely the output of the TTCM decoders rather than that of the MMSE MUD, is supplied for the GA MUD. This reliable improvement therefore offers a better starting point for the GA's search. Secondly, the iterative processing ensures that the detected $L$-user symbol vector can be optimized in two dimensions, as demonstrated in Figure 3. During every iteration, on one hand, each $L$-symbol vector at a specified subcarrier slot is optimized by the GA in the context of the user domain. On the other hand, the entire TTCM-coded frame of each user is optimized by the TTCM decoder in the context of the TTCM-related codeword domain, or more specifically the frequency domain. Therefore, as the iterative processing continues, an information exchange takes place between the two domains and thus an improved system performance may be expected.

\section{IMPROVED MUTATION SCHEME}

The GA-based MUDs [6-8] invoke an intelligent natural evolution-like process to find the optimum ML solution by searching through a small subset of the typically excessive $\mathrm{ML}$ search space. At the commencement of the GA-based search, an initial GA population consisting of $X$ number of so-called individuals is created. One of these initial individuals is generated by making a hard decision at the output of the MMSE MUD, while the other $(X-1)$ individuals are generated by mutating the MMSE solution. An individual is represented by a symbol vector containing $L$ complex-valued symbols, each of which belongs to one of the $L$ number of users at the specific subcarrier considered. More specifically, the $i^{t h}$ individual of the $y^{t h}$ generation is expressed as:

$$
\tilde{\mathbf{s}}_{i}^{(y)}=\left[\tilde{s}_{i, 1}^{(y)}, \tilde{s}_{i, 2}^{(y)}, \ldots, \tilde{s}_{i, L}^{(y)}\right], i=\{1, \ldots, X\}
$$

where $\tilde{s}_{i, l}^{(y)}$ denotes the $l^{t h}(l=1, \ldots, L)$ gene of the $i^{\text {th }}$ individual. Each gene can be represented by any element of $\mathcal{M}_{c}$, where $\mathcal{M}_{c}$ is the set containing the $2^{m}$ number of legitimate modulated symbols, while $m$ denotes the number of bits per symbol. The population consisting of $X$ number of individuals then forms the starting point of the optimization process, which is referred to as the $y=0^{t h}$ generation. Various genetic operators, such as selection, cross-over, mutation [6], etc. are then invoked for the sake of evolving the GA's population from one generation to the next, until the predefined maximum GA generation index of $Y$ is reached. For reasons of space economy, the interested reader is referred to [6-8] for more details of the GA-based detection.

In the mutation operation invoked during the GA-based search process, any gene of an individual may be mutated to another legitimate gene under the control of the specific mutation strategy employed. The efficiency of the GA's mutation scheme is important for the success of 
the entire evolution procedure, since it provides a chance for the individuals of the current population to influence the forthcoming ones, so that new areas of the total search space may be explored, thus increasing the chances of finding the optimum solution.

\subsection{Conventional Uniform Mutation}

The so-called Uniform Mutation (UM) [6-8] has been widely used in the GA-based MUD literature. According to the UM, when a gene is subjected to mutation, it will be substituted by a different symbol in $\mathcal{M}_{c}$ based on a uniform mutation-induced transition probability $p_{m t}^{(i j)}$, which quantifies the probability of the $i^{\text {th }}$ legitimate symbol becoming the $j^{t h}$. For the sake of brevity, from now on we refer to this probability as the transition probability. However, this fixed uniform transition probability fails to reflect the realistic channel conditions that the system is subjected to. More specifically, when considering a specific received symbol, the adjacent constellation symbols are more likely to be the transmitted symbol, than the more distant ones. Hence, it may be more reasonable to consider only the neighbouring symbols as the potential mutation candidates, and assign a modified biased transition probability, which is dependent on both the Euclidean distance from the original symbol and on the Signal-to-Noise Ratio (SNR). In other words, the GA's search space may be substantially reduced with the aid of a biased mutation, which pays less attention to the constellation points that are far from the received symbol, and thus increasing the GA's efficiency.

\subsection{Biased $Q$-function Based Mutation}

In this section, a novel mutation scheme will be presented, which we shall refer to as Biased Q-function Based Mutation (BQM). According to $\mathrm{BQM}$, for an original gene to be mutated, an SNR-related biased transition probability $p_{m t}^{(i j)}$ will be assigned to each of the target candidate symbols in $\mathcal{M}_{c}$. The calculation of $p_{m t}^{(i j)}$ may be carried out with the aid of the widely-known $Q$-function [9]:

$$
Q(x)=\frac{1}{\sqrt{2 \pi}} \int_{x}^{\infty} e^{-t^{2} / 2} d t, \quad x \geq 0
$$

Figure 4: Illustration of the 1D transition probability $p_{m t}^{(i j)}$ for 4QAM.

For the sake of easy explanation, let us first consider a simple onedimensional (1D) scenario. In Figure 4 we plotted the 1D real component of the constellation symbols $\hat{s}_{i}^{(l)}$ in the context of the 4QAM modem constellation. The horizontal axis is then divided into two zones, each of which represents one specific 1D constellation symbol $s_{R i}(i=1, \ldots, 2)$, as separated by the vertical dashed line of Figure 4 . If $s_{R 1}$ is the original gene to be mutated, the Gaussian distribution $N(0, \sigma)$ may be centered at the position of $s_{R 1}$, where $\sigma$ is the noise variance at a given SNR level. In this specific example, $s_{R 2}$ is the only mutation target and the $1 \mathrm{D}$ transition probability of mutating from $s_{R 1}$ to $s_{R 2}$, i.e. $p_{m t}^{(12)}$, is characterized by the shadow area shown in Figure 4, which is given by:

$$
p_{m t}^{(12)}=Q\left(\frac{d_{0}}{\sigma}\right)
$$

where $d_{0}$ is half of the distance between the neighbouring constellation symbols. Similarly, we have $p_{m t}^{(21)}=Q\left(\frac{d_{0}}{\sigma}\right)$. Furthermore, we also
Figure 5: Illustration of the 2D transition probability $p_{m t}^{(i j)}$ for $4 \mathrm{QAM}$, which is the product of the relevant 1D transition probabilities. $s_{R i}$ and $s_{I i}(i=1,2)$ denote the 1D constellation symbols in the context of the real and imaginary components of the 4QAM constellation symbols, respectively.

have a certain probability for the original gene to remain unchanged, which can also be expressed as: $p_{m t}^{(11)}=p_{m t}^{(22)}=1-Q\left(\frac{d_{0}}{\sigma}\right)$. Hence, the corresponding two-dimensional (2D) symbol transition probability $p_{m t}^{(i j)}$ can be derived by combining the $1 \mathrm{D}$ real and imaginary transition probabilities. For the specific 1D-based 4QAM example of Figure 4, we plot the corresponding $2 \mathrm{D}$ constellation in Figure 5 . In Figure 5, for instance, the 2D transition probability of mutating from the constellation symbol $\hat{s}_{1}^{(l)}$ to $\hat{s}_{2}^{(l)}$, namely $p_{m t}^{(12)}$, can be calculated by multiplying the two relevant $1 \mathrm{D}$ transition probabilities according to:

$$
p_{m t}^{(12)}=p_{m t}^{\left(\frac{11}{m}\right)} \cdot p_{m t}^{\left(\frac{12}{m t}\right)}=\left(1-Q\left(\frac{d_{0}}{\sigma}\right)\right) \cdot Q\left(\frac{d_{0}}{\sigma}\right),
$$

while the associated $2 \mathrm{D}$ probability of remaining in the current state is $p_{m t}^{(11)}=p_{m t}^{(11)} \cdot p_{m t}^{(11)}=\left(1-Q\left(\frac{d_{0}}{\sigma}\right)\right)^{2}$. Note that when a mutation takes place, the mutating gene should not be allowed to be mutated to itself. In order to remove the effect of the probability of mutating a symbol to itself, the 2D transition probability $p_{m t}^{(i j)}(i \neq j)$ should be normalized with $p_{m t}^{(i i)}$ by following the principles of conditional probability theory $[10]$. Note that the proposed BQM scheme can be readily extended to $M$-dimensional (MD) constellations, since the MD transition probability associated with a specific MD symbol can be readily derived upon multiplying the $M$ number of corresponding $1 \mathrm{D}$ transition probabilities. It is worth pointing out that the proposed BQM scheme only requires a modest "once-for-all" calculation, since we can derive the associated transition probabilities by offline precomputation for typical SNR levels, which can then be stored in the BS's memory for reuse.

\subsection{Simplified BQM}

Furthermore, the proposed BQM scheme can be effectively simplified, when only a subset of all the theoretically-possible mutation target symbols are considered. More precisely, for the original gene subjected to mutation, we may only consider its adjacent neighbouring constellation symbols as mutation target candidates, since the original transmitted symbol is less unlikely to be corrupted to a relatively distant constellation symbol. An example of the simplified BQM designed for 16QAM is provided in Figure 6. As shown in Figure 6, for example, we assume that $\hat{s}_{1}^{(l)}$ is the original gene subjected to mutation, and $\hat{s}_{i}^{(l)}(i=2, \ldots, 9)$ represents the adjacent neighbours of $\hat{s}_{1}^{(l)}$. Moreover, the GA's search space can be further reduced, when we only consider the nearest neighbours of $\hat{s}_{1}^{(l)}$ as the legitimate mutation candidates, namely the symbols $\hat{s}_{i}^{(l)}(i=3,5,6,8)$, which are printed in grey in Figure 6 . Each of these symbols is then assigned an equal 2D transition probability $p_{m t}^{(1 j)}=1 / 4(j=3,5,6,8)$, while all other constellation symbols printed in white are neglected. In this case, the BQM scheme is simplified to a new scheme similar to UM, which we may refer to as the Closest-Neighbour Uniform Mutation (CNUM) scheme. Note that in CNUM the corresponding transition probability value is only dependent on the location of the original gene. Thus, it is unnecessary to calculate the CNUM transition probabilities, which are summarized in Table 1. Hence, by introducing the simplified BQM 
scheme or the CNUM arrangement, the computational complexity of $\mathrm{BQM}$ can be significantly reduced.

Figure 6: An example of the simplified BQM for 16QAM.

\begin{tabular}{|c|c|}
\hline Modem & Transition probability value set \\
\hline 4QAM & $1 / 2$ \\
\hline 16QAM & $1 / 2,1 / 3,1 / 4$ \\
\hline 64QAM & $1 / 2,1 / 3,1 / 4$ \\
\hline
\end{tabular}

Table 1: Possible transition probability values for the CNUM scheme.

\section{SIMULATION RESULTS}

In this section, we characterize the performance of the proposed TTCMaided SDMA-OFDM system using the BQM-aided MMSE-IGA MUD. The simulation results were obtained using a $4 Q A M$ scheme communicating over a channel characterized by the 3-path Short Wireless Asynchronous Transfer Mode (SWATM) CIR given on page 78 of [1], assuming that the channels' transfer functions were perfectly known. The maximum path delay $\tau_{\max }$ is $48.9 \mathrm{~ns}$, while each of the paths experiences independent Rayleigh fading having the same normalized Doppler frequency of $f_{d}^{\prime}=1.235 \times 10^{-5}$. The OFDM modem employed $K=512$ subcarriers and 64 samples as the cyclic prefix. For the iterative TTCM scheme [3] used, the code memory $\nu$ was fixed to 3 , the generator polynomial expressed in octal format was $\left[\begin{array}{ll}13 & 6\end{array}\right]$, and the TTCM codeword length as well as channel interleaver depth were fixed to 1024 symbols. For each of the various schemes evaluated, a similar total number of TTCM decoding iterations was maintained, so that the total TTCM-related complexity remained approximately the same for all systems.

Figure 7 compares the Bit Error Rate (BER) performance achieved by the non-iterative GA aided schemes of $[7,8]$ to that of the proposed IGA-aided systems. The performance of the TTCM-assisted MMSESDMA-OFDM system, the TTCM-assisted optimum ML-detected system and the uncoded single-user scheme employing a single receiver when communicating over an AWGN channel are also provided for reference, respectively. The numbers in the round brackets seen in the legends of Figure 7 denote the associated number of IGA MUD iterations and the total GA or $\mathrm{ML}$ complexity ${ }^{1}$, respectively. As shown in Figure 7, at the same GA complexity, the IGA MUD assisted systems outperformed their non-iterative GA MUD aided counterparts, regardless of the different mutation schemes used. This is because the proposed IGA MUD's initial population was generated from the output of the TTCM decoders, which is more reliable than that of the MMSE MUD of $[7,8]$. On the other hand, the BQM-aided systems achieved a better performance than the UM-aided schemes, since BQM is more efficient in guiding the GA towards the optimum solution. Furthermore, the system employing the BQM-aided two-iteration IGA MUD was ca-

\footnotetext{
${ }^{1}$ Here the complexity is quantified in terms of the number of optimization metric computations required by the GA or the ML detection process $[7,8]$
}

Figure 7: BER versus $E_{b} / N_{0}$ performance comparison of the iterative or non-iterative TTCM-assisted MMSE-GA-SDMA-OFDM system using $\mathbf{U M}$ or BQM, while employing a 4QAM scheme for transmission over the SWATM channel, where $\mathbf{L}=\mathbf{6}$ users are supported with the aid of $\mathbf{P}=\mathbf{6}$ receiver antenna elements, respectively.

pable of achieving a virtually indistinguishable performance from that of the optimum ML-aided system, despite its significantly lower complexity of 200 metric evaluations.

Figure 8: BER versus $E_{b} / N_{0}$ performance comparison of the iterative or non-iterative TTCM-assisted MMSE-GA-SDMA-OFDM system using $\mathbf{U M}$ or BQM, while employing a 16QAM scheme for transmission over the SWATM channel, where $\mathbf{L}=\mathbf{6}$ users are supported with the aid of $\mathbf{P}=\mathbf{6}$ receiver antenna elements, respectively.

Moreover, when a high-throughput modem such as 16QAM is employed, BQM may significantly outperform UM. Figure 8 shows that the UM-aided scheme yielded a high error floor, even when the IGA was employed. By contrast, BQM significantly improved the performance by lowering the error floor by at least two orders of magnitude. Furthermore, when the number of IGA MUD iterations was increased, the performance of the BQM-aided system was dramatically improved, while the UM-aided scheme still suffered from an error floor. This suggests that the BQM-aided scheme is capable of exploiting the benefits arising from both a better initial GA population and from a higher number of IGA MUD iterations.

The left-hand side of Figure 9 shows the $E_{b} / N_{0}$ gain achieved by the BQM-IGA assisted TTCM-MMSE-SDMA-OFDM systems employing 16QAM at the BER of $10^{-5}$, while using different number of IGA MUD iterations. In this scenario, six users were supported by six receiver antennas. The $E_{b} / N_{0}$ gain is defined here as the $E_{b} / N_{0}$ dif- 
Figure 9: $\mathbf{E}_{\mathbf{b}} / \mathbf{N}_{0}$ Gain at the BER of $10^{-5}$ versus the number of IGA MUD iterations (left) and the number of users (right) performance of the TTCM-assisted MMSE-IGA-SDMA-OFDM system using BQM, while employing a 16QAM scheme for transmission over the SWATM channel, where $L=6$ (left) and $L=4,5,6$ (right) users are supported with the aid of $\mathbf{P}=\mathbf{6}$ receiver antenna elements, respectively.

ference measured at the BER of $10^{-5}$ between the systems employing the BQM-IGA MUD or dispensing with its employment. As expected, when we had a higher number of IGA MUD iterations, a higher $E_{b} / N_{0}$ gain was observed. Furthermore, a higher gain can be attained by employing a higher GA population size.

The right-hand side of Figure 9 exhibits the corresponding $E_{b} / N_{0}$ gains achieved by the SDMA-OFDM system exploiting $P=6$ receiver antenna elements at the BER of $10^{-5}$, in the scenarios where the number of users varies from four to six. It is shown that when the user load becomes higher, a higher gain can be attained. For example, for the single-iteration IGA-aided system, a further gain of about $3.5 \mathrm{~dB}$ is achieved, when the number of users increases from four to six. Secondly, a higher number of IGA MUD iterations provides a higher gain for the system. For instance, in the fully-loaded scenario, namely for $L=P=6$, the two-iteration IGA-aided system achieves a further gain of about $2.7 \mathrm{~dB}$ over its single-iteration counterpart.

Explicitly, using a higher number of IGA MUD iterations results in a further increased complexity, although this may still be significantly lower than that of the ML-aided scheme, which imposed an excessive complexity and hence cannot be simulated. For example, in the scenario where the 16QAM modem was employed in the sixuser system, the associated complexity of the ML-aided scheme is as high as $16^{6}=16,777,216$. By contrast, the two-iteration BQM-IGA MUD associated with $X=40$ and $Y=5$ imposes a modest complexity of 400 , which is only $0.00238 \%$ of the excessive ML-related complexity, while achieving a considerable $E_{b} / N_{0}$ gain of about $6 \mathrm{~dB}$ over the base-line TTCM-MMSE-SDMA-OFDM benchmarker system at the BER of $10^{-5}$, as observed at the right-hand side of Figure 9.

As discussed in Section 3.3, the BQM scheme may be simplified to the CNUM arrangement, which reduces the complexity of BQM. However, CNUM does not dramatically degrade the system's performance. Figure 10 provides a comparison of CNUM and BQM for both low- and high-throughput systems. As observed in Figure 10, the BQM-GA-assisted system achieved a slightly better performance than its CNUM-GA-assisted counterpart. This suggests that in such scenarios CNUM may become an attractive alternative to $B Q M$ for the sake of further decreasing the complexity imposed.

\section{CONCLUSIONS}

From the investigations conducted, we conclude that the TTCM-aided SDMA-OFDM system using the proposed MMSE-IGA MUD is capable of achieving a similar performance to that of the optimum MLassisted TTCM-SDMA-OFDM arrangement. Furthermore, this is attained at a significantly lower computational complexity than that imposed by the ML-assisted system, especially when the number of users is high. Moreover, the novel BQM scheme is capable of improving the GA's efficiency, thus significantly increasing the chances of finding the
Figure 10: BER versus $E_{b} / N_{0}$ performance comparison of the TTCM-assisted MMSE-GA-SDMA-OFDM system using CNUM or BQM, while employing a 4QAM or 16QAM scheme for transmission over the SWATM channel, where $\mathbf{L}=\mathbf{6}$ users are supported with the aid of $\mathbf{P}=\mathbf{6}$ receiver antenna elements, respectively.

optimum GA solution in high-SNR and/or high-throughput scenarios. Our simulation results show that the scheme that combines BQM with the IGA MUD yields the best performance in all the scenarios considered. For example, as evidenced by Figures 7 and 8 , an $E_{b} / N_{0}$ gain of about $6 \mathrm{~dB}$ was achieved by the proposed BQM-IGA-aided scheme in comparison to the MMSE-SDMA-OFDM benchmarker scheme, both in the scenarios where 4QAM and 16QAM was employed.

\section{REFERENCES}

[1] L. Hanzo, M. Münster, B. J. Choi, and T. Keller, $O F D M$ and $M C$ CDMA for Broadband Multi-user Communications, WLANs and Broadcasting. IEEE Press - John Wiley \& Sons Ltd., 2003.

[2] S. Verdu, Multiuser Detection. Cambridge University Press, 1998.

[3] L. Hanzo, T. H. Liew, and B. L. Yeap, Turbo Coding, Turbo Equalisation and Space-Time Coding for Transmission Over Fading Channels. New York, USA: IEEE Press - John Wiley \& Sons Ltd., 2002.

[4] M. Jiang, S. X. Ng, and L. Hanzo, "TCM, TTCM, BICM and BICM-ID Assisted MMSE Multi-User Detected SDMA-OFDM Using Walsh-Hadamard Spreading," in Proceedings of IEEE Vehicular Technology Conference '04 Spring, (Milan, Italy), pp. 1129-1133, May 17-19 2004.

[5] D. E. Goldberg, Genetic Algorithms in Search, Optimization, and Machine Learning. Reading, Massachusetts: Addison-Wesley, 1989.

[6] L. Hanzo, L.-L. Yang, E.-L. Kuan, and K. Yen, Single- and MultiCarrier DS-CDMA: Multi-User Detection, Space-Time Spreading, Synchronisation and Standards. IEEE Press - John Wiley \& Sons Ltd., 2003.

[7] M. Jiang and L. Hanzo, "Improved Hybrid MMSE Detection for Turbo Trellis Coded Modulation Assisted Multi-User OFDM Systems," IEE Electronics Letters, vol. 40, pp. 1002-1003, August 2004.

[8] M. Jiang and L. Hanzo, "Genetically Enhanced TTCM Assisted MMSE Multi-User Detection for SDMA-OFDM," in Proceedings of IEEE Vehicular Technology Conference '04 Fall, (Los Angeles, USA), September 26-29 2004.

[9] J. G. Proakis, Digital Communications. McGraw-Hill International Edition, 4th ed., 2001.

[10] W. Ledermann and E. Lloyd, Handbook of Applicable Mathematics, Volume II: Probability, vol. 2. John Wiley \& Sons Ltd., 1980. 